\title{
Preclinical development of tumor-infiltrating lymphocyte therapy for ovarian cancer
}

\author{
Donastas Sakellariou-Thompson ${ }^{{ }^{*}}$, Cara Haymaker ${ }^{1}$, Marie-Andree Forget ${ }^{1}$, Amir Jazaeri ${ }^{2}$, Patrick Hwu', \\ Chantale Bernatchez ${ }^{1}$
}

From 30th Annual Meeting and Associated Programs of the Society for Immunotherapy of Cancer (SITC 2015) National Harbor, MD, USA. 4-8 November 2015

\section{Background}

Immunotherapy has become an effective cancer therapy, particularly in the case of checkpoint blockade and adoptive $\mathrm{T}$ cell therapy (ACT). ACT exploits the presence of tumor-infiltrating lymphocytes (TIL) by exponentially expanding their numbers ex vivo and re-infusing them into the patient in an autologous setting. With the effectiveness of TIL therapy already well established in multiple Phase II studies in melanoma, there is a push to translate it to other malignancies such as ovarian cancer $(\mathrm{OvCa})$ [1].

\section{Methods}

The presence of TIL is correlated with greatly increased survival in OvCa $[2,3]$ suggesting that TIL effectively control the disease and provide a rationale to test TIL therapy in this setting. To assess the feasibility, we characterized the immune component of $\mathrm{OvCa}$, explored the ability to grow \& expand TIL from tumor fragments, and tested their functionality.

\section{Results}

Extensive flow cytometry analysis detected a robust, activated $\mathrm{T}$ cell infiltrate that can be grown from OvCa samples obtained pre- and post-chemotherapy. The addition of an agonistic anti-41BB antibody to the cultures preferentially increased CD8 ${ }^{+}$TIL outgrowth as well as favored the expansion of NK cells. Importantly, success rate of TIL growth was increased from $40 \%$ to $90 \%$ for cultures grown without and with anti-41BB respectively. It was established next that the $\mathrm{CD}^{+}$TIL initially grown with anti-41BB could be rapidly expanded at least 1000 fold over two weeks. Finally, the rapidly expanded T cells exhibited anti-tumor capabilities in the context of redirected killing assays.

\section{Conclusions}

In conclusion, further flow cytometry analysis to identify other agonistic and inhibitory targets is needed along with additional in vitro and in vivo experiments. However, the initial data suggest that TIL therapy for OvCa could be a viable therapeutic option in the future.

\section{Authors' details}

'The Department of Melanoma Medical Oncology, The University of Texas MD Anderson Cancer Center, Houston TX, USA. ${ }^{2}$ The Department of Gynecologic Oncology and Reproductive Medicine, The University of Texas MD Anderson Cancer Center, Houston TX, USA.

Published: 4 November 2015

\section{References}

1. Santoiemma PP, Powell DJ: Tumor infiltrating lymphocytes in ovarian cancer. Canc Biol Ther 2015, 16:807-820.

2. Milne K, Kobel M, Kalloger SE, Barnes RO, Gao D, Gilks CV, et al: Systematic analysis of immune infiltrates in high-grade serious ovarian cancers reveals CD20, FoxP3, and TIA-1 as positive prognostic factors. PLoS One 2009, 4:e6412.

3. Nielsen JS, Sahota RA, Milne K, Kost SE, Nesslinger NJ, Watson PH, et al: CD20+ tumor-infiltrating lymphocytes have an atypical CD27-memory phenotype and together with CD8+ T cells promote favorable prognosis in ovarian cancer. Clin Cancer Res 2012, 18(12):3281-3292.

\section{doi:10.1186/2051-1426-3-S2-P48}

Cite this article as: Sakellariou-Thompson et al:: Preclinical development of tumor-infiltrating lymphocyte therapy for ovarian cancer. Journal for ImmunoTherapy of Cancer 2015 3(Suppl 2):P48. 